\title{
Visualizing Patterns of Medication Switching Among Major Depressive Patients with Various Stability and Difficulty to Treatments
}

\author{
Yu-Chun Hung' \\ Hsi-Chung Chen (iD ${ }^{2}$ \\ Po-Hsiu Kuo iD ${ }^{2,3}$ \\ Mong-Liang Lu id ${ }^{4,5}$ \\ Ming-Chyi Huang ${ }^{6}$ \\ Chun-Hsin Chen ${ }^{4,5}$ \\ Sabrina Wang ${ }^{7}$ \\ Wei-Chung Mao ${ }^{8}$ \\ Chang-Shiann $\mathrm{Wu}^{9}$ \\ Tzu-Hua Wu (iD) 1,5,10,11 \\ 'Division of Clinical Pharmacy, School of \\ Pharmacy, College of Pharmacy, Taipei Medical \\ University, Taipei, I I0, Taiwan; ${ }^{2}$ Department of \\ Psychiatry, National Taiwan University Hospital, \\ Taipei, Taiwan; ${ }^{3}$ Department of Public Health \& \\ Institute of Epidemiology and Preventive \\ Medicine, College of Public Health, National \\ Taiwan University, Taipei, Taiwan; ${ }^{4}$ Department \\ of Psychiatry, Wan Fang Hospital \& School of \\ Medicine, College of Medicine, Taipei Medical \\ University, Taipei, Taiwan; ${ }^{5}$ Psychiatric Research \\ Center, Wan Fang Hospital, Taipei Medical \\ University, Taipei, Taiwan; ${ }^{6}$ Department of \\ Psychiatry, Taipei City Hospital, Songde Branch, \\ Taipei, Taiwan; ${ }^{7}$ Institute of Anatomy and Cell \\ Biology, School of Medicine, National Yang-Ming \\ University, Taipei, Taiwan; ${ }^{8}$ Department of \\ Psychiatry, Cheng-Hsin General Hospital, \\ Taipei, Taiwan; 'Department of Information \\ Management, College of Management, National \\ Formosa University, Huwei Township, Yunlin \\ County, Taiwan; ${ }^{10} \mathrm{Ph}$.D. Program in Drug \\ Discovery and Development Industry, College \\ of Pharmacy, Taipei Medical University, Taipei, \\ Taiwan; "Master Program in Clinical \\ Pharmacogenomics and Pharmacoproteomics, \\ College of Pharmacy, Taipei Medical University, \\ Taipei, Taiwan:
}

Correspondence: Tzu-Hua Wu Division of Clinical Pharmacy, School of Pharmacy, College of Pharmacy, Taipei Medical University, 250 Wu-Hsing Street, Taipei, I I0, Taiwan

Tel +886-22736I66I ext. 6172

Email thwu@tmu.edu.tw
Introduction: Efforts have been made in assessing efficacy and tolerability to various antidepressants, but understanding personalized chances of stability to medication switching sequence is still inconclusive. This study aimed to identify naturalistic switching patterns of medication in stratifying MDD patients.

Methods: MDD patients were stratified based on treatment difficulty evaluated with the "Treatment Resistance to Antidepressants Evaluation Scale for Unipolar Depression" (TRADES). The duration of the time of diagnoses until the final switch to another class of antidepressants was used as prediction of unstable to drug therapy. ROC analysis was used to determine the cutoff values. A continuous temporal events function from the visual analytic tool was employed to perform patterns of switching between distinct pharmacological class such as selective serotonin reuptake inhibitors (SSRIs) and serotonin-norepinephrine reuptake inhibitors (SNRIs).

Results: TRADES scores of 4.5 and not-switching times of 12.5 months were used as cutoff values to divide patients into four subgroups: stable/easy-to-treat (SE), unstable/easy-to-treat (UE), stable/difficult-to-treat (SD) and unstable/difficult-to-treat (UD). A total of $80 \%$ and $76.9 \%$ of patients initially treated with the SSRIs paroxetine or fluoxetine, respectively, were predicted to be stable to drug therapy. Approximately $70 \%, 44.8 \%$ and $41.4 \%$ of patients initially treated with the SNRIs fluvoxamine, sertraline and venlafaxine, respectively, were predicted to be UD, and $60 \%$ of patients using duloxetine were predicted to be stable to drug therapy. Analysis of the switching phenomenon showed that SSRIs were the first prescribed medications and mostly taken by the stable subgroups, and SNRIs were the preferentially chosen switching alternative. Medication switching patterns in unstable MDD patients are discussed.

Conclusion: Paroxetine, fluoxetine and duloxetine users were mostly stable among MDD patients in Taiwan with various stability and difficulty to treatments. Although responsiveness to specific medication sequence is likely required for clinical application, the results provide a baseline for such studies.

Keywords: visualization, major depressive disorder, patterns of medication switching, antidepressants, stability

\section{Introduction}

Globally, more than 264 million people of all ages suffer from depression. Major depressive disorder (MDD) is a disabling chronic mental health illness. Up to twothirds of patients with major unipolar depression will not respond to the first medication prescribed. ${ }^{1}$ In fact, an inadequate response may appear after two consecutive antidepressant treatments with adequate dosage and adequate duration 
with antidepressants from different pharmacological classes. Lack of early improvement, defined by a $<20 \%$ reduction in depression rating scale scores at 2-4 weeks, may be predictive of eventual clinical nonresponse to an antidepressant. Therefore, early switching strategies with antidepressants were suggested even though there was limited quality evidence. ${ }^{2}$ Therefore, developing strategies to improve the life-long disease control of those MDD patients who did not continuously receive adequate therapy $^{3}$ is an urgent need. ${ }^{4}$

Recent meta-analysis suggests that newer antidepressants are similar in efficacy and overall tolerability. ${ }^{5}$ Efforts to identify achievement of a stable antidepressant regimen from electronic medication records in patients with MDD were also approached by Hughes et al. ${ }^{6}$ The emerging consensus within the Asia-Pacific region further defined the adequate duration of two antidepressant therapies given at adequate doses was 6-8 weeks during a major depressive episode. ${ }^{7}$ Even though there is still substantial heterogeneity that exists to identify difficulty to treat patients, ${ }^{8}$ most clinical guidelines recommend switching to another antidepressant as a first-line strategy for nonresponders, although augmentation and combination strategies also have evidence to support their use. ${ }^{2}$ However, switching between different pharmacological classes of antidepressants may or may not show superiority over continuation of the original antidepressant treatment. ${ }^{9}$

To monitor continuality of medication therapy for patients with chronic diseases, a medication review ${ }^{10}$ on clinical outcomes is needed to ensure that patients can access the appropriate medications ${ }^{11}$ and treatment guidelines. ${ }^{12}$ Our previous study ${ }^{13}$ developed a new, multidimensional, clinometric instrument, the Treatment Resistance to Antidepressants Evaluation Scale (TRADES), to evaluate the level of treatment difficulty with antidepressants in MDD patients. Total TRADES scores were also associated with a higher utilization of psychiatric outpatient and admission services before the index date. Furthermore, it is thought that total TRADES scores could predict a higher number of visits to psychiatric outpatient, emergency, and admission services following the index date. Therefore, the TRADES could help to quantify the level of treatment difficulty with antidepressants in major depressive disorder and predict severity of symptoms by BDI-II. ${ }^{13}$

Based on therapeutic drug monitoring, ${ }^{14}$ blood drug levels combined with patients' symptom scores can be used as pharmacotherapy outcome predictions. Switching medications were also recommended by pharmacotherapy guidelines as a strategy to manage non-response in MDD patients. In fact, in our previous study,$^{13}$ duration of stability on drug therapy was also used as a factor, but not class of medications, contributing level of treatment difficulty. This study considered the timing at which doctors stopped switching medications as a discrimination factor to see patients' stable treatment response to drug therapy. Instead of using drug levels to define the minimum threshold levels to predict outcomes, this study employed TRADES scores to define the minimum threshold levels of treatment difficulty with the timing without switching to distinct pharmacological class of antidepressants to predict patients' stable response to drug therapy. A visualization analysis was applied to facilitate performing naturalistic prescribing patterns including switching sequences for stratified patients according to scores of treatment difficulty and the time to find suitable medications indicated by how long it takes to stop switching drugs between different classes of antidepressants. Strategies for antidepressant pharmacotherapies and timing sequences of medication selection for MDD patients with various levels of treatment difficulty are reported.

\section{Methods}

The participants were similar to those described in our previous record-based study, ${ }^{13}$ approved by the committees of the National Taiwan University Hospital, WanFang Hospital and Taipei City Hospital, Songde Branch, for developing the TRADES scoring system. Written informed consent was obtained from each participant and all methods were performed in accordance with the Declaration of Helsinki. Clinicians changed medications based on diagnosis and their personal judgements, experiences and patients' preferences. The lower the patient's TRADES score was, the easier the patient was to treat. TRADE itself can be a predictor for the lifetime disease control. The current study was additionally approved by the research ethics committees of the Taipei Medical University-Joint Institutional Review Board (N201703085), and employed TRADES (assessed at index date) together with the timing of stabilization to drug therapy on certain class of pharmacology as shown in Figure 1. The number of recruited patients was initially 107 , and only those patients $(\mathrm{n}=97)$ whose records were continuously documented for two years were then further enrolled in the current study unless patients were 

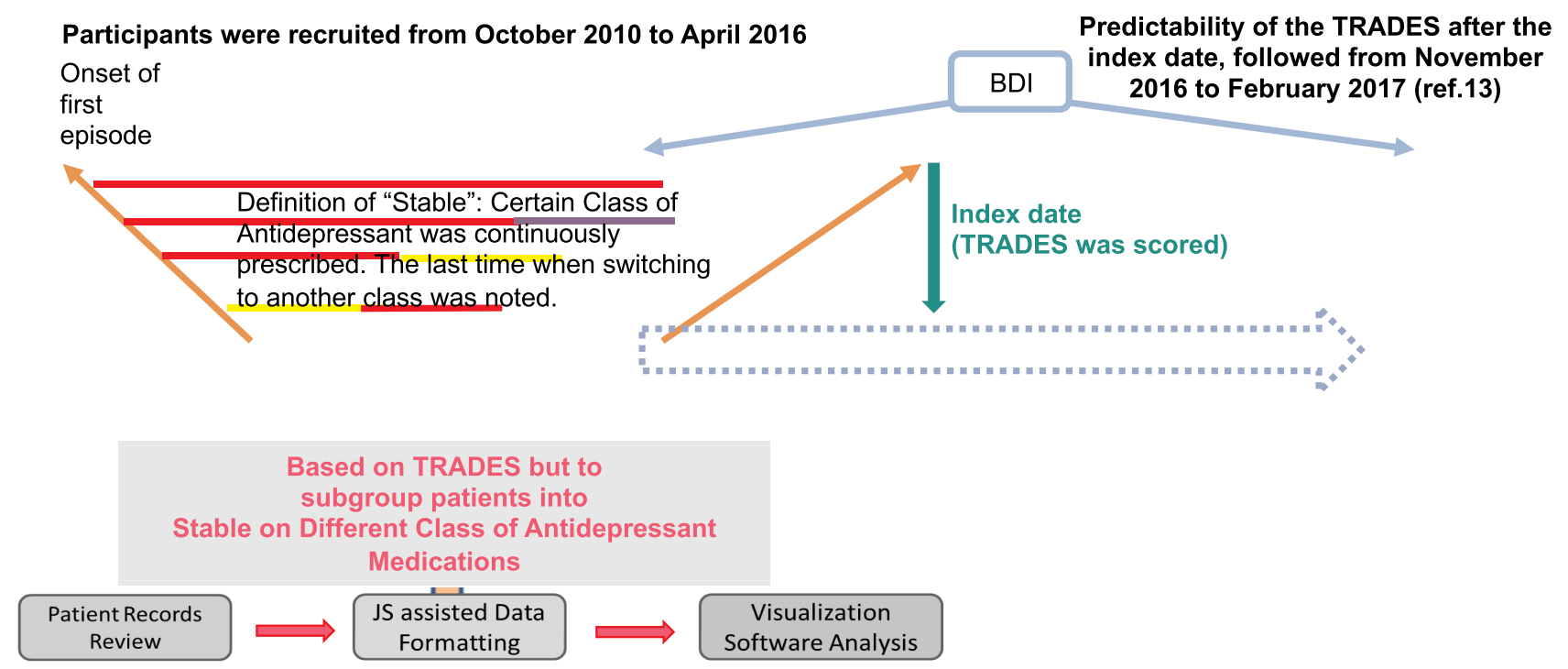

Figure I Flow chart of this study including patient record review and data formatting and then analysis by visualization software. Current assessment on the medication switching patterns for response of stability to drug therapy and TRADES score assigned to the patient at the index date were based on the two-year medical records. Prescriptions patterns were performed by visualization analysis.

confirmed to be remitted within two years. The pharmacological classes of medications prescribed for these patients included tricyclic antidepressants (TCAs), selective serotonin reuptake inhibitors (SSRIs), serotoninnorepinephrine reuptake inhibitors (SNRIs), serotonin antagonists and reuptake inhibitors (SARIs), reversible inhibitors of monoamine oxidase-A (RIMA), norepinephrine-dopamine reuptake inhibitors (NDRIs) and noradrenergic and specific serotonergic antidepressants (NaSSAs) and antipsychotic medications.

SPSS software was used for the receiver operating characteristic (ROC) curve analysis to define cutoff values to differentiate patients by TRADES scores (indicating treatment difficulty) and the time needed before a doctor re-prescribed the same class of medications as previous one (indicating stable to treatment) without considering augmentation medications. The shorter the time until a patient's prescription medication was not switched between pharmacological classes was, the earlier that patient's MDD status was considered stable to treatments.

The visualization analysis utilized EventFlow software (Version 2.3.4), which was kindly provided by the HumanComputer Interaction Lab (University of Maryland, US), with Microsoft Windows 7 SP2 (64-bit) and Java SE Version 8 Update 121 (64-bit). After aligning the data based on the date when MDD was diagnosed, different pharmacological classes of prescribed medications for the recruited patients over 2 years were displayed by different colors. Based on the time- dependent sequences, the patients were grouped together based on the initial medication and similar prescription patterns and ranked based on these similarities by EventFlow. The visualization analysis shows the cumulated results for prescription patterns based on the sequence and duration. Based on the first event (first prescribed medication), EventFlow shows the continuously occurring second event based on the second most prescribed medication or pharmacological class. The same principle is used in subsequent analyses.

\section{Results}

\section{Clinical Characteristics and Evaluation of Recruited Patients}

This study included a total of 97 MDD patients, and their two-year medical records after diagnosis with MDD were subjected to analysis. A total of $78.4 \%$ were female; the mean age was $52.9 \pm 13.2$ years; and the mean age of onset was $44.4 \pm 13.5$ years. The percentages of antidepressant medication use and utilization of augmentation medications are shown in Tables 1 and 2. As shown in Tables 1, 59/97 patients were prescribed SSRIs during this study period; among these patients, the rank order was as follows: sertraline $(29 ; 49.2 \%)>$ paroxetine $(17$; $28.8 \%)>$ fluoxetine $(13 ; 22.0 \%)>$ escitalopram $(8 ; 13.6 \%)$ $>$ fluvoxamine (3; 5.1\%). Among SNRI users (44/97), the rank order was as follows: venlafaxine $(33 ; 75.0 \%)>$ duloxetine $(15 ; 34.1 \%)$. Among TCA users $(5 / 97)$, the 
Table I Comparisons Across Pharmacological Classes of Antidepressant Medications Prescribed to the Study Patients. The Numbers of Patients Prescribed a Medication in Each Pharmacological Class and the Particular Medications in Each Pharmacological Class

\begin{tabular}{|c|c|c|}
\hline $\begin{array}{l}\text { Pharmacological } \\
\text { Class of } \\
\text { Antidepressants }\end{array}$ & $\begin{array}{l}\text { Medication } \\
\text { Name }\end{array}$ & $\begin{array}{l}\text { Number of Patients }(\%)^{\S} \\
\text { Using the Medication } \\
\text { During the Study Period }\end{array}$ \\
\hline SSRI & $\begin{array}{l}\text { Escitalopram } \\
\text { Fluoxetine } \\
\text { Fluvoxamine } \\
\text { Paroxetine } \\
\text { Sertraline }\end{array}$ & $\begin{array}{cc}59(60.8 \%) & \\
& 8 \\
& 13 \\
& 3 \\
17 \\
29\end{array}$ \\
\hline SNRI & $\begin{array}{l}\text { Duloxetine } \\
\text { Venlafaxine }\end{array}$ & $\begin{array}{ll}44(45.4 \%) & \\
& 15 \\
& 33\end{array}$ \\
\hline TCA & $\begin{array}{l}\text { Doxepin } \\
\text { Imipramine }\end{array}$ & $5(5.2 \%)$ \\
\hline SARI & Trazodone & $4(4.1 \%)$ \\
\hline RIMA & Moclobemide & $2(2.1 \%)$ \\
\hline NDRI & Bupropion & $\begin{array}{ll}17(17.5 \%) & \\
& 17\end{array}$ \\
\hline NaSSA & Mirtazapine & $\begin{array}{ll}18(18.6 \%) & \\
& 18\end{array}$ \\
\hline
\end{tabular}

Note: ${ }^{\S}$ patients may be prescribed with more than one antidepressant.

Abbreviations: SSRI, selective serotonin reuptake inhibitor; SNRI, serotoninnorepinephrine reuptake inhibitor; TCA, tricyclic antidepressant; SARI, serotonin2 antagonist reuptake inhibitor; RIMA, reversible inhibitor of monoamine oxidase-A (MAO-A); NDRI, noradrenergic-dopamine reuptake inhibitor; NaSSA, noradrenergic-specific serotonergic antidepressants.

rank order was as follows: imipramine $(4 ; 80.0 \%)>$ doxepin $(1 ; 20.0 \%)$. The prescribed medications in other pharmacological classes included trazodone (SARI), moclobemide (RIMA), bupropion (NDRI) and mirtazapine (NaSSA).

Among the 97 recruited patients, 38 utilized augmentation medications. The most prescribed class of medications was atypical antipsychotics (21, 55.3\%; quetiapine: 20, $95.2 \%)$, followed by traditional antipsychotics (18, 21.1\%; sulpiride: $17,94.4 \%$ ). The others included mood stabilizers, stimulants, thyroid agents, and the numbers of patients utilizing these medications are summarized in Table 2.

The demographics, "Treatment Resistance to Antidepressants Evaluation Scale for Unipolar Depression"
Table 2 Comparisons Across Pharmacological Classes of the Augmentation Medications to the Study Patients ( $n=38 ; 39.2 \%)$ Who Was Prescribed with Augmentation Medications. The Numbers of Patients Prescribed a Medication in Each Pharmacological Class and the Particular Medication in Each Pharmacological Class

\begin{tabular}{|c|c|c|}
\hline $\begin{array}{l}\text { Pharmacological } \\
\text { Class of } \\
\text { Augmentation } \\
\text { Medications }\end{array}$ & $\begin{array}{l}\text { Medication } \\
\text { Name }\end{array}$ & $\begin{array}{l}\text { Number of Patients } \\
(\%)^{\S} \text { Using the } \\
\text { Medication During } \\
\text { the Study Period }\end{array}$ \\
\hline Atypical antipsychotic & $\begin{array}{l}\text { Amisulpride } \\
\text { Olanzapine } \\
\text { Quetiapine } \\
\text { Risperidone } \\
\text { Zotepine }\end{array}$ & $\begin{array}{r}21 \text { (55.3\%) } \\
\text { । } \\
4 \\
20 \\
2 \\
\text { I }\end{array}$ \\
\hline Mood stabilizer & $\begin{array}{l}\text { Carbamazepine } \\
\text { Lamotrigine } \\
\text { Lithium } \\
\text { Valproic acid }\end{array}$ & $\begin{array}{ll}4(10.5 \%) & \\
& 1 \\
& 1 \\
& 1 \\
& 1\end{array}$ \\
\hline Psychostimulant & Methylphenidate & $\begin{array}{ll}2(5.3 \%) & \\
& 2\end{array}$ \\
\hline Typical antipsychotic & $\begin{array}{l}\text { Chlorpromazine } \\
\text { Sulpiride } \\
\text { Trifluoperazine }\end{array}$ & $\begin{array}{r}18(21.1 \%) \\
1 \\
17 \\
1\end{array}$ \\
\hline Thyroid preparation & Levothyroxine & I $(2.6 \%)$ \\
\hline
\end{tabular}

Notes: ${ }^{\S}$ patients may be prescribed with more than one medication as augmentation.

(TRADES-UD is abbreviated here as TRADES) scores and diagnosed diseases or remission status for the patients are summarized as Supplementary Data 1-1 and 1-2. During the study period, the number of remitted patients was 11 $(11.3 \%)$, and 62 had comorbidities. Of those diagnosed with comorbidities, $69.4 \%$ (43/62) were diagnosed with the other mental illness, including 69.8\% (30/43) with generalized anxiety disorder and $27.9 \%$ (12/43) with panic disorder.

\section{ROC Analysis and Outcome Prediction}

According to the ROC analysis results (Figure 2), TRADES $>4.5$ was true when the time that the prescription medication class no longer changed was 12.5 months after MDD diagnosis (sensitivity plus specificity $=1.383$; area under the ROC curve $=0.702$ ). Therefore, patients whose final switch in prescribed medication class occurring earlier than 12.5 months while TRADES $\leq 4$ were predicted to be stable/easy- 


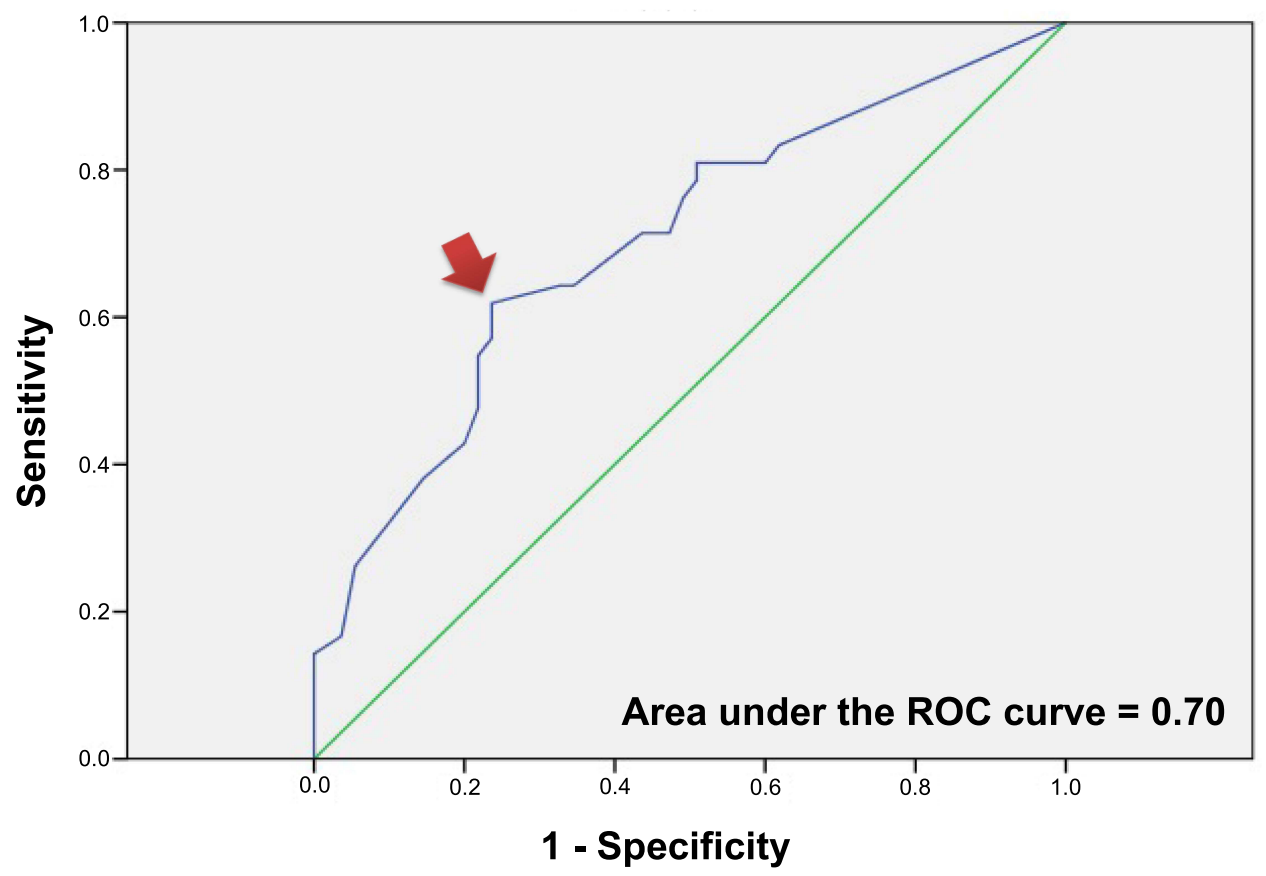

Figure 2 ROC analysis results. The arrow points to the cutoff value for TRADE scores larger than 4.5 (considered difficult-to-treat) while the true condition is indicated at I 2.5 months. Patients with TRADES scores larger than 4.5 and the final switch in prescribed antidepressant medication between different pharmacological class occurring later than 12.5 months were predicted to be unstable/difficult-to-treat.

to-treat (SE; $n=42)$. Patients whose final switch in prescribed medication class occurring later than 12.5 months while TRADES $\leq 4$ were predicted to be unstable/easy-to-treat (UE; $n=13$ ). Patients whose final switch in prescribed medication class occurring earlier than 12.5 months while TRADES $>5$ were predicted to be stable/difficult-to-treat /responsive (SD; $n=16)$. Patients whose final switch in prescribed medication class occurring later than 12.5 months while TRADES $\geq 5$ were predicted to be unstable/difficult-to -treat (UD; $n=26)$.

\section{Visualization Analysis for Medication Timing and Sequence Patterns}

In Figure 3, the most and the first prescribed pharmacological class of medication was SSRIs (in red; $n=35$ ), followed by SNRIs (in yellow; $n=24$ ), which were ranked as the 2nd most frequently used medication. The other types of prescriptions were SSRIs combined with augmentation drug (in red combined with orange; $n=9$ ), NaSSAs alone (bright green; $\mathrm{n}=9$ ), SNRIs with augmentation drug (in yellow combined with orange; $n=5$ ), NDRIs (in purple; $\mathrm{n}=3$ ), NDRIs with augmentation drug (in purple combined with orange; $n=3)$, RIMAs $(n=1)$ and other classes $(n=4)$.

The pharmacological class most often chosen as the second prescribed medication for patients was SNRIs.
In general, patients who were first prescribed SSRIs were then switched to SNRIs.

Figure 4 shows prescription visualization patterns of four groups of patients based on the ROC predictions: stable/easy-to-treat (SE), unstable/easy-to-treat (UE), stable/difficult-to-treat (SD) and unstable/difficult-totreat (UD). The patterns of prescriptions looked similar for both the SE and SD groups regardless of whether their TRADES score categorized them as easy or difficult-to-treat. The patterns of prescribed pharmacological classes were more complicated, especially in the UD group. Surprisingly, the mostly first prescribed medications were SNRIs (mean duration of uses: $18.9 \pm 7.7$ months) and most patients $(85.7 \%)$ used venlafaxine. In contrast, SSRIs were first prescribed in patients categorized into the $\mathrm{SE}, \mathrm{UE}$, and $\mathrm{SD}$ subgroups with a range of mean durations of uses $(18.7 \pm 8.4,13.5 \pm 7.4$, and $20.9 \pm$ 7.8 months, respectively). The most commonly chosen SSRI drugs for the SE, UE, and SD subgroups were fluoxetine $(27.2 \%)$ /paroxetine $(27.2 \%)$, escitalopram $(40.0 \%)$, and fluoxetine $(45.5 \%)$, respectively.

To differentiate the prescribing patterns among the TRADES groups, we further identified the frequency of utilization for specific drugs in the subgroups. SSRI drugs, as shown in Supplementary Data 2, such as paroxetine or fluoxetine, tended to be prescribed for stable response 
A

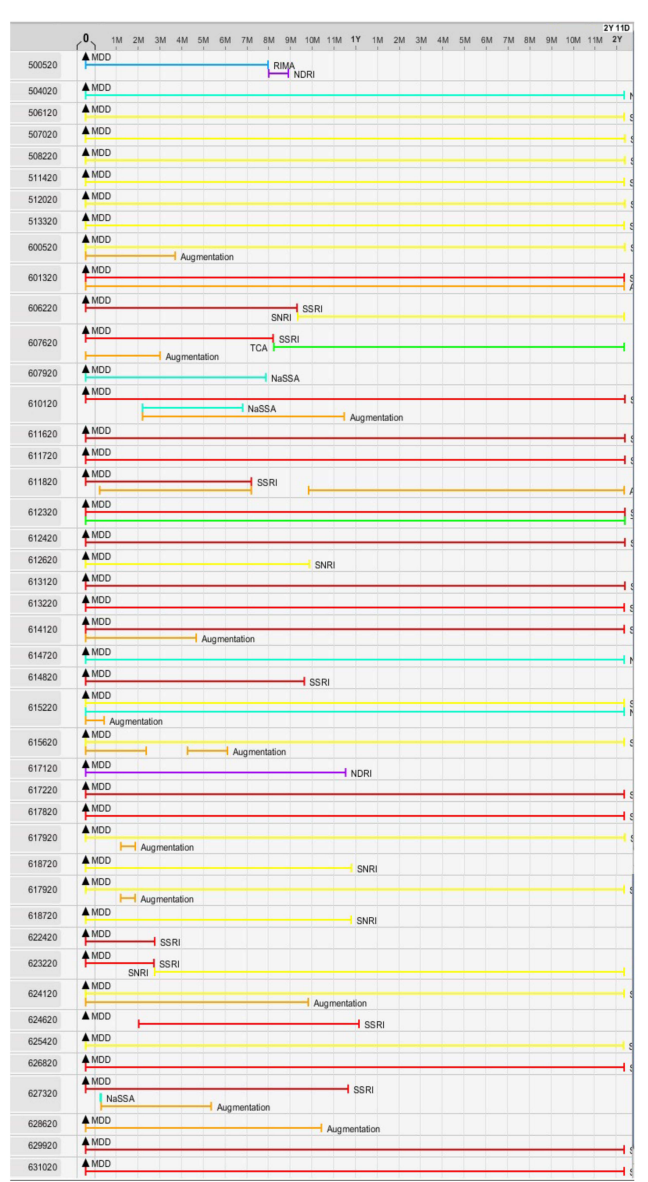

C

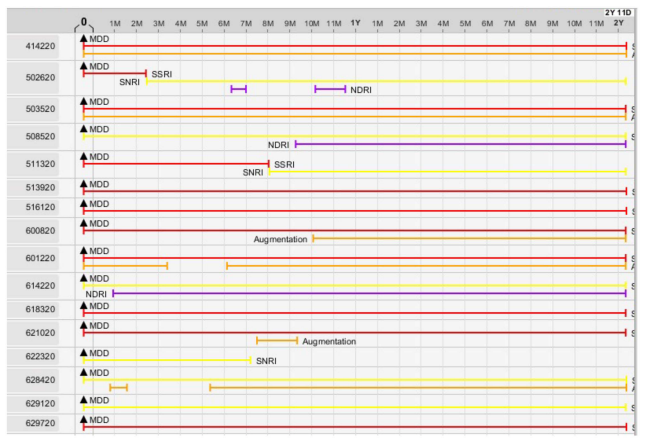

B

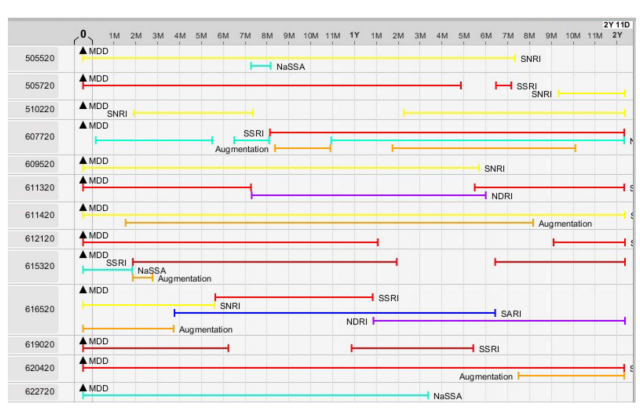

D

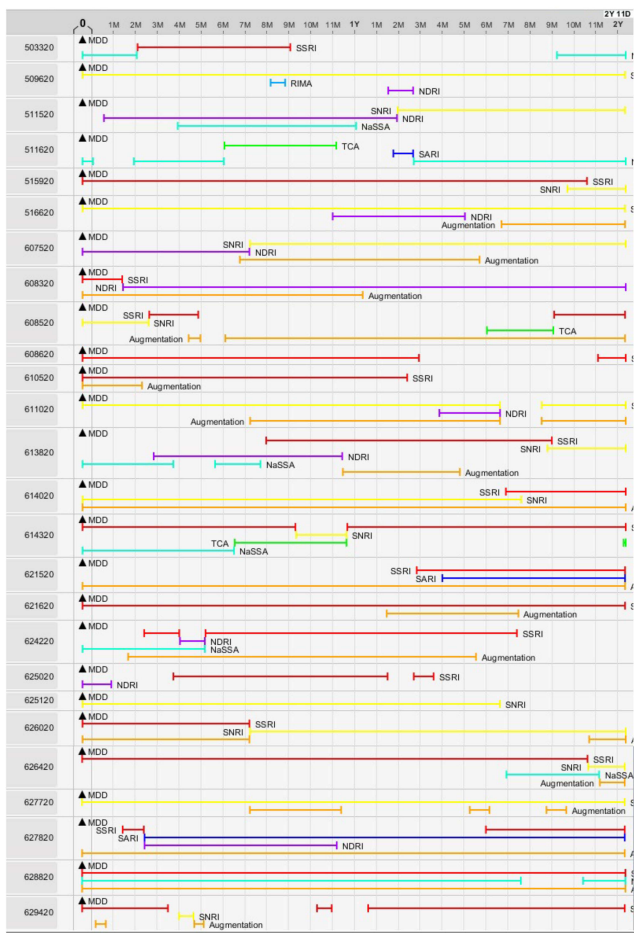

Figure 3 MDD participant ( $n=97$ ) categorized into four groups ((A) SE; (B) UE; (C) SD; (D) UD) and their individual prescription information for each pharmacological class of medication and respective time frame was presented by color bar using visualization software.

Abbreviations: SE, stable/easy-to-treat; UE, unstable/easy-to-treat; SD, stable/difficult-to-treat; UD, unstable/difficult-to-treat. 


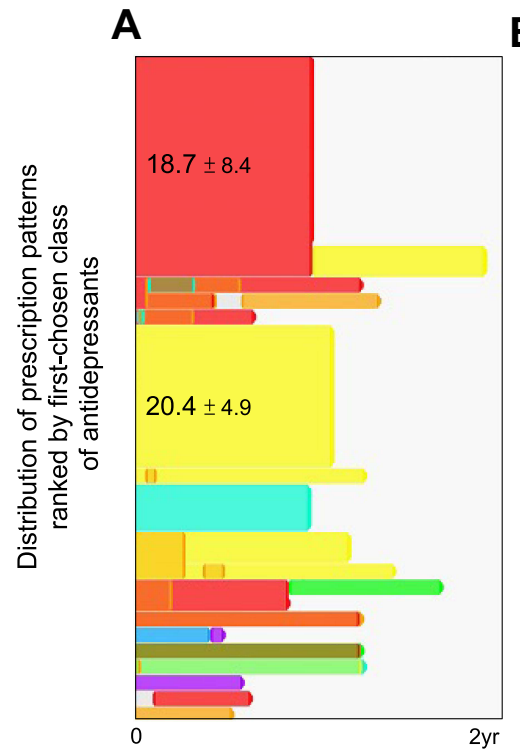

B

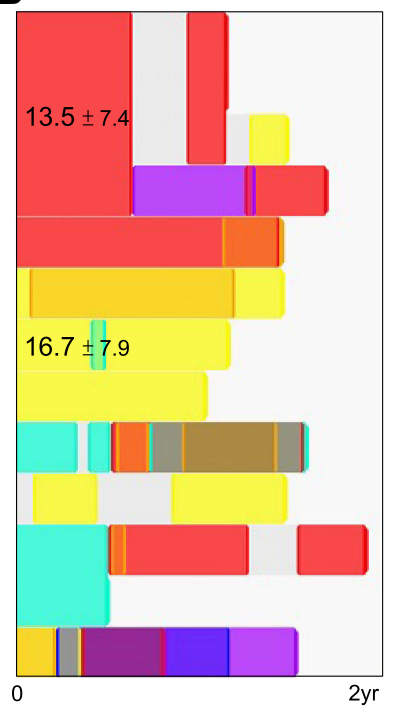

C

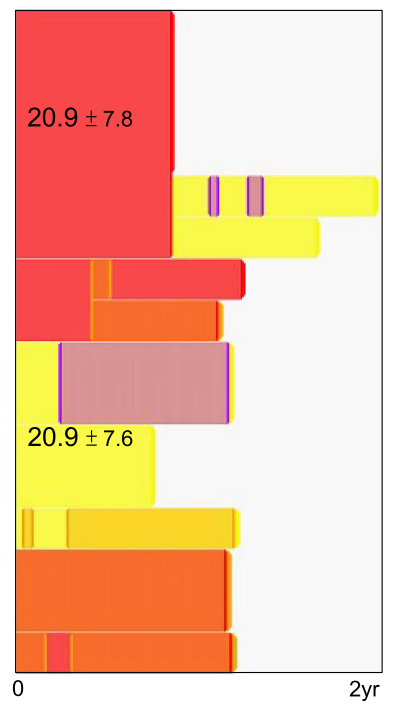

D

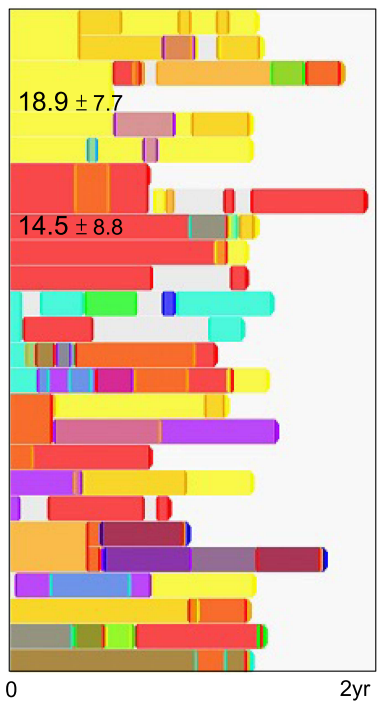

Duration of treatments (months)

$\Delta$ MDD $\square$ SSRI $\square$ SNRI $\square$ TCA $\square$ SARI $\square$ RIMA $\square$ NDRI $\square$ NaSSA $\square$ Augmentation

Figure 4 The preferred first-chosen pharmacological class, preferred medications and switching patterns were categorized into four groups ((A) SE; (B) UE; (C) SD; (D) UD). Mostly used prescription patterns was ranked from the top of each figure and each distinct pharmacological class was illustrated by different colors. The mean duration of top two first-chosen class of antidepressants was indicated for each subgroup of patients and expressed in months \pm standard deviation.

Abbreviations: SE, stable/easy-to-treat; UE, unstable/easy-to-treat; SD, stable/difficult-to-treat; UD, unstable/difficult-to-treat.

patients (SE and SD). Escitalopram tended to be prescribed for patients categorized as easy-to-treat (SE and UE), who may or may not have shown a stable response. Fluvoxamine or sertraline was chosen for patients in the UD group. SNRI drugs, as shown in Supplementary Data $\underline{3}$, such as duloxetine, tended to be used in the difficult-totreat group. Among that prescribed venlafaxine, approximately $40 \%$ were categorized into the UD group.

Regarding the patterns of switching medications, SSRIs were initially prescribed in the SE and SD subgroups, and these patients were preferentially switched to SNRIs. SSRIs were initially prescribed in the UE subgroup and were preferentially switched to NDRIs. SNRIs were initially prescribed in the UD subgroup and were preferentially switched to SSRIs.

\section{Add-on or Augmentation with Antipsychotic Medications}

Twenty-one patients (ie, 4, 4, 3, and 10 patients in the SE, UE, SD, and UD groups, respectively) were prescribed antipsychotics. During the switching of antidepressant therapies, the mean duration until an add-on antipsychotic medication prescription occurred was $0.98,7.8,6.1$, and 9.4 months in the SE, UE, SD, and UD subgroups, respectively. The summary of the patterns of prescriptions is shown in Supplementary Data 4, and the drugs quetiapine or sulpiride as add-on or augmentation were mostly prescribed to patients categorized into the SD or UD subgroups. Among responders who were initially treated with SSRIs as the first antidepressant, the mean durations that they were continuously prescribed antipsychotics were 7.1 $(\mathrm{n}=3), 8.0(\mathrm{n}=2), 4.8(\mathrm{n}=1)$, and $2.5(\mathrm{n}=3)$ months in the SE, UE, SD, and UD subgroups, respectively. One of the remitted patients was prescribed an antipsychotic at 0.3 months after treatment with SSRIs, and the augmentation was continued for 5.1 months.

\section{Discussion}

Current study was based on first two-year records and applied the different patterns of temporal switching between different pharmacological classes of antidepressants in patients to discriminate unstable on drug therapy of selection and difficult to treat scores (TRADES) to subgroup patients. Patients who had a final switch between pharmacological classes in less than 12.5 months $($ AUROC $=0.702)$ after starting pharmacotherapy and TRADES scores that were less than 4.5 were considered as stable and easy-to-treat depressive subgroups. Overall, after MDD was diagnosed, the first drug prescribed for 
patients was mostly SSRIs $(\mathrm{n}=47 ; 48.5 \%)$, and the most commonly used drug was sertraline $(\mathrm{n}=16 ; \mathrm{S}) ; 80 \%$ of those patients who were initially treated with paroxetine or $76.9 \%$ of those initially treated with fluoxetine were predicted to have a stable response (SE or SD). Moreover, 30 patients $(30.9 \%)$ were prescribed SNRIs as their first antidepressant after MDD was diagnosed; the most commonly used drug was venlafaxine $(\mathrm{n}=22 ; \mathrm{V})$, and $60 \%$ of patients using duloxetine were predicted to have a stable response (SE or $\mathrm{SD})$.

Having depression patients achieve remission has always been the goal of depression treatment, but studies have shown that most patients do not achieve this goal, and $25-50 \%$ of patients who responded to treatment still have depression-related symptoms. ${ }^{15}$ This study found that UD patients, ie, unstable/difficult-to-treat patients, accounted for $16.5 \%$ (16/97) of the sample, which was lower than earlier reports.

Among the 97 depression patients in this study, 11 patients $(11.3 \%)$ achieved remission within the two-year treatment period after MDD diagnosis. After subgrouping by the current criteria obtained from the ROC analysis, 55 patients were considered easy-to-treat regardless of stability, and nearly one-fifth of the patients (18.2\%) achieved remission. The numbers of remitted patients were $8,2,0$, and 1 in the SE (total subgroup $n=42$ ), UE (total subgroup $\mathrm{n}=13$ ), SD (total subgroup $\mathrm{n}=16$ ), and UD (total subgroup $\mathrm{n}=26$ ) subgroups, respectively. Among those predicted to be stable response patients $(n=58), 13.8 \%(8 / 58)$ remitted, which was a lower rate than earlier reports ${ }^{16}$ suggesting that $20-40 \%$ of stable response patients can achieve remission.

Based on the percentages of patients initially prescribed a certain pharmacological class, the ranking was as follows: SSRI> SNRI> NaSSA (12.4\%), NDRI (3.1\%), TCA (1.0\%), RIMA (1.0\%) and SARI (0\%). This is consistent with a previous study in which patients were diagnosed with depressive disorder and were generally given SSRIs as first-line antidepressants. ${ }^{17}$ The durations that the drugs in each pharmacological class was administered were $17.7 \pm 8.4$ months for SSRIs, $20.6 \pm 6.5$ months for SNRIs, and 11.3 $\pm 9.6,6.5 \pm 5.3,24.0$, and 8.0 months for the other pharmacological categories (NaSSA, NDRI, TCA, and RIMA, respectively). The highest frequencies for SSRI and SNRI prescriptions were sertraline (49.2\%) and venlafaxine $(75 \%)$, respectively.

The rationale ${ }^{18,19}$ to prescribe venlafaxine and duloxetine, both of which are SNRI antidepressants, is supported by findings that both were associated with higher remission rates; however, $70 \%$ or $41.4 \%$ of patients who were initially treated with fluvoxamine or venlafaxine (both belong to the SNRI class), respectively, were predicted to be UD patients. Therefore, venlafaxine treatment may result in better outcomes as the first choice of medication than fluvoxamine. Moreover, the observed duration of treatment with SNRIs was long and followed by SSRIs, which was consistent with a previous study. ${ }^{20}$ Interestingly, the remission rate associated with the use of venlafaxine $(45 \%)$ was better than that with SSRIs $(35 \%){ }^{18,21}$ Moreover, the results of Henssler et $\mathrm{al}^{22}$ also supported that combining a reuptake inhibitor with an antagonist of presynaptic $\alpha 2$-autoreceptors such as the NaSSA drug mirtazapine seems to be significantly more effective than other combinations.

Furthermore, regardless of pharmacological class, the percentages of the initial prescribed drug for the 97 MDD patients were venlafaxine $(22.7 \%)$, sertraline $(16.5 \%)$, and mirtazapine $(12.4 \%)$. In this study, after the diagnosis of MDD, more than half (55.7\%) of the patients were initially treated with either mirtazapine, escitalopram, venlafaxine, or sertraline. This prescribing behavior is consistent with the drugs that potentially benefit patients based on depression scale score improvements $(>50 \%)$, ie, mirtazapine, escitalopram, venlafaxine, and sertraline ${ }^{23}$ performed better than duloxetine, fluoxetine, fluvoxamine, paroxetine, or reboxetine.

Differential switching patterns among antidepressants in the four subgroups of MDD patients (SE, UE, SD, UD) with various TRADES and stability to drug therapy were characterized by Visualization Software. In terms of pharmacological classes, switching from SSRIs to SNRIs occurred more frequently in the patients who were subgrouped as SE and SD. The SSRIs prescribed in the UE group were more frequently switched to NDRIs. After patients were treated with SSRIs, the differential switching patterns among various subgroups showed that NDRIs were the top selection in the SE, SD, and UD subgroups, while SNRIs were the top selection in the UE subgroup, as shown in Figure 4. Moreover, SNRIs ( $n=4 ; 26.67 \%)$ firstly prescribed in the UD group $(n=15)$ were more frequently $(n=3)$ switched to SSRIs and two of those stable on SSRIs at least within the two-year records. After any patients were treated with SNRI, the differential switching patterns among various subgroups was that NDRIs were selected in the UE subgroup $(n=1)$, while there were no consistent patterns for SE or SD subgroups. 
Among those patients categorized as SE, only one patient was prescribed moclobemide after MDD was diagnosed and continuously up to 8 months without interruption; then, the patient was switched to an NDRI (bupropion) for one month and reached remission within one year after MDD was diagnosed. The limited clinical use of RIMAs, such as moclobemide, was also described in an earlier study $^{24}$ because of serotonin syndrome and hypertensive crisis. $^{25,26}$ In fact, $21 \%$ of TRD patients ${ }^{27}$ treated with RIMA drugs that are combined with other antidepressant drugs showed significant improvement in their condition without complications. Therefore, combination therapies with RIMA drugs with close follow-up for potential adverse drug reactions and risks due to drug-drug or food (tyramine)-drug interactions by experienced psychiatric teams may be considered for refractory patients who cannot achieve a treatment response or are considered UD patients. Therefore, combination treatments for patients treated with moclobemide who are predicted to be UD are warranted.

Moreover, there was one remitted patient who was prescribed the NDRI bupropion during the study period for one month and then switched to sertraline at the fourth month after MDD was diagnosed. It has been reported that bupropion ${ }^{28}$ can be used alone or in combination with other antidepressants since it has similar effectiveness compared with other antidepressant drugs and is well tolerated with little sexual dysfunction or weight gain and less sedation. ${ }^{29}$ Therefore, switching to bupropion has been suggested if patients with depression have problems with weight gain or sexual dysfunction. NDRIs were also suggested to be prescribed together with SNRIs to improve outcomes. In the current study, as shown in Supplementary Data 5, more than half of those patients treated with NDRIs (17 patients. $17.5 \%$; 2 in the ES group, 2 in the UE group, 3 in the SD group, and 10 in the UD group) were predicted to be in a difficult-to-treat subgroup (SD or UD) and were prescribed a combination treatment with an SNRI. However, it is noteworthy that the NDRI class has the shortest duration of treatment of any class - which likely stems from bupropion as the singular agent of the class.

Augmentation with a second-generation antipsychotic (SGA) or lithium is another strategy to obtain greater improvements in depressive scores since the remission rates were $89.3 \%$ and $86.2 \%$, which were higher than prescription switching between classical antidepressants or antidepressant combinations. ${ }^{30}$ As shown in Supplementary Data 6, there were 20 depression patients $(20.6 \% ; 3,3,2$, and 12 patients predicted to be in the SE,
UE, SD, and UD subgroups, respectively) who had been treated with the additive treatment drug quetiapine. Among the remitted patients in the ER group, 2 patients were treated with sertraline, and 1 patient was treated with a combination including quetiapine after 9 days. Moreover, there were 17 depression patients $(17.5 \%$; 6 , 1, 4, 6 patients predicted to be in the SE, UE, SD, and UD subgroups, respectively) who had been treated with the additive treatment drug sulpiride, and in nearly half of those patients in each subgroup, it was combined with an SSRI, as seen in the Supplementary Data 7. Understanding the decision and the timing for adding antipsychotics to the antidepressant treatment regimen may provide an explanation for those patients with better disease control.

Beck Depression Inventory-II (BDI-II) scores were evaluated and collected after the study and the percentage of outcome prediction matched with patients' individual BDI scores considered normal was $68.9 \%$ for the easy-totreat subgroups. The percentage of outcome prediction matched with patients' individual BDI scores considered an indication of severely depressed was $43.2 \%$ for the difficult-to-treat subgroups. Since the patients did not receive regular evaluations of their depression scores, such as the BDI (Supplementary Data 8), this study cannot further elaborate on improvements in MDD symptoms.

\section{Limitations}

This study also focusses on the naturalistic prescribing behaviors of doctors incorporated with hospital medication availability. Since three study hospitals may not have the same antidepressants within the same class available to prescribe for patients; therefore, this study defined the switching medication as changes between different classes of pharmacology. In fact, patterns indicative of stability might also contribute to a higher TRADES score and some patients who do not attain clinical response or remission may also be stable with treatment in the real-world. The patients recruited in the current study were assumed to have $100 \%$ compliance with the prescribed medications even though we did not count whether their drug pills were taken before each visit. It has been reported that patients with depression have lower compliance and persistence, which is believed to be related to the pharmacological category of antidepressant drugs. ${ }^{20}$ For example, patients treated with SNRIs seem to have higher compliance and persistence than those treated with SSRIs, and the other pharmacological classes of antidepressant drugs have similar low levels of compliance/persistence to treatments as SSRIs. 


\section{Conclusion}

This study utilized program-assisted data preprocessing to efficiently differentiate and visualize the naturalistic prescription-switching phenomenon in Taiwan with various stability and difficulty to treatments. SSRIs (16/47 patients used sertraline) and SNRIs (22/30 patients used venlafaxine) were the top two initially prescribed classes. Paroxetine or fluoxetine and duloxetine users mostly were stable MDD patients. The prescription medicationswitching phenomenon showed that SSRIs were initially prescribed mostly for the SE and SD subgroups, SNRIs were preferentially chosen as an alternative, and early addon/augmentation with antipsychotics may improve outcomes in the unstable and difficulty to treat MDD subpopulation.

\section{Acknowledgment}

We appreciate the Human-Computer Interaction Lab at the University of Maryland for creating the EventFlow software and granting us a noncommercial license.

\section{Disclosure}

The authors report no conflicts of interest in this work.

\section{References}

1. Little A. Treatment-resistant depression. Am Fam Physician. 2009;2:167-172.

2. Kudlow PA, McIntyre RS, Lam RW. Early switching strategies in antidepressant non-responders: current evidence and future research directions. CNS Drugs. 2014;7:601-609. doi:10.1007/s40263-0140171-5

3. Souery D, Mendlewicz J. Compliance and therapeutic issues in resistant depression. Int Clin Psychopharmacol. 1998;13:S13-8. doi:10.1097/00004850-199802002-00003

4. Reutfors J, Andersson TM, Tanskanen A. et al. Risk factors for suicide and suicide attempts among patients with treatment-resistant depression: nested case-control study. Arch Suicide Res;2019. 1-15. doi:10.1080/13811118.2019.1691692

5. Cipriani A, Furukawa TA, Salanti G, et al. Comparative efficacy and acceptability of 21 antidepressant drugs for the acute treatment of adults with major depressive disorder: a systematic review and network meta-analysis. Lancet. 2018;391(10128):1357-1366. doi:10.1016/S0140-6736(17)32802-7

6. Hughes MC, Pradier MF, Ross AS. Assessment of a prediction model for antidepressant treatment stability using supervised topic models. JAMA Netw Open. 2020;3(5):e205308. doi:10.1001/ jamanetworkopen.2020.5308

7. $\mathrm{Ng} \mathrm{CH}$, Kato T, Han C, et al. Definition of treatment-resistant depression - Asia Pacific perspectives. J Affect Disord. 2019;245:626-636. doi:10.1016/j.jad.2018.11.038

8. Brown S, Rittenbach K, Cheung S, McKean G, MacMaster FP, Clement F. Current and common definitions of treatment-resistant depression: findings from a systematic review and qualitative interviews. Can J Psychiatry. 2019;6:380-387. doi:10.1177/ 0706743719828965
9. Bartova L, Dold M, Kautzky A, et al. Results of the European Group for the Study of Resistant Depression (GSRD) - basis for further research and clinical practice. World $j$ Biol Psychiatry. 2019;6:427-448. doi:10.1080/15622975.2019.1635270

10. Lehnbom EC, Stewart MJ, Manias E, Westbrook JI. Impact of medication reconciliation and review on clinical outcomes. Ann Pharmacother. 2014;1542-6270.

11. Patel E, Pevnick JM, Kennelty KA. Pharmacists and medication reconciliation: a review of recent literature. Integrated Pharm Res Practice. 2019; Volume 8:39-45. doi:10.2147/IPRP.S169727

12. Taylor RW, Marwood L, Oprea E, et al. Pharmacological augmentation in unipolar depression: a guide to the guidelines. Int $j$ Neuropsychopharmacol. 2020;9:587-625.

13. Liu TY, Kuo PH, Lu ML, et al. Quantifying the level of difficulty to treat major depressive disorder with antidepressants: treatment resistance to antidepressants evaluation scale. PLoS One. 2020;1: e0227614. doi:10.1371/journal.pone.0227614

14. Lu ML, Wu YX, Chen CH, et al. Application of plasma levels of olanzapine and n-desmethyl-olanzapine to monitor clinical efficacy in patients with schizophrenia. PLoS One. 2016;2:e148539. doi:10.1371/journal.pone.0148539

15. Nierenberg AA, Keefe BR, Leslie VC, et al. Residual symptoms in depressed patients who respond acutely to fluoxetine. $J$ Clin Psychiatry. 1999;4:221-225. doi:10.4088/JCP.v60n0403

16. Kennedy N, Paykel ES. Residual symptoms at remission from depression: impact on long-term outcome. J Affect Disord. 2004;2-3:135-144. doi:10.1016/S0165-0327(03)00054-5

17. Wells BG, Schwinghammer TL, DiPiro CV. Pharmacotherapy Handbook. 9 ed. McGraw-Hill; 2014.

18. Thase ME. Effectiveness of antidepressants: comparative remission rates. J Clin Psychiatry. 2003;3-7.

19. Van Rhoads RG. Treating depression to remission: target recovery, and give patients back their lives. Curr Psychiatr. 2005;9:15-28.

20. Keyloun KR, Hansen RN, Hepp Z, Gillard P, Thase ME, Devine EB. Adherence and persistence across antidepressant therapeutic classes: a retrospective claims analysis among insured us patients with major depressive disorder (mdd). CNS Drugs. 2017;5:421-432. doi:10.1007/s40263-017-0417-0

21. Thase ME, Entsuah AR, Rudolph RL. Remission rates during treatment with venlafaxine or selective serotonin reuptake inhibitors. Br j Psychiatry. 2001;178(3):234-241. doi:10.1192/ bjp.178.3.234

22. Henssler J, Bschor T, Baethge C. Combining antidepressants in acute treatment of depression: a meta-analysis of 38 studies including 4511 patients. Can J Psychiatry. 2016;1:29-43. doi:10.1177/ 0706743715620411

23. Cipriani A, Furukawa TA, Salanti G, et al. Comparative efficacy and acceptability of 12 new-generation antidepressants: a multiple-treatments meta-analysis. Lancet. 2009;9665:746-758. doi:10.1016/S0140-6736(09)60046-5

24. Shulman KI, Herrmann N, Walker SE. Current place of monoamine oxidase inhibitors in the treatment of depression. CNS Drugs. 2013;10:789-797. doi:10.1007/s40263-013-0097-3

25. Krishnan KR. Revisiting monoamine oxidase inhibitors. J Clin Psychiatry. 2007;68 Suppl 8:35-41.

26. Dunkley EJ, Isbister GK, Sibbritt D, Dawson AH, Whyte IM. The hunter serotonin toxicity criteria: simple and accurate diagnostic decision rules for serotonin toxicity. QJM. 2003;9:635-642. doi:10.1093/qjmed/hcg109

27. Thomas SJ, Shin M, McInnis MG, Bostwick JR. Combination therapy with monoamine oxidase inhibitors and other antidepressants or stimulants: strategies for the management of treatment-resistant depression. Pharmacotherapy. 2015;4:433-449. doi:10.1002/ phar. 1576 
28. Patel K, Allen S, Haque MN, Angelescu I, Baumeister D, Tracy DK. Bupropion: a systematic review and meta-analysis of effectiveness as an antidepressant. Therapeutic Adv Psychopharmacol. 2016;2:99-144. doi:10.1177/2045125316629071

29. Dhillon S, Yang LP, Curran MP. Spotlight on bupropion in major depressive disorder. CNS Drugs. 2008;7:613-617. doi:10.2165/ 00023210-200822070-00006
30. Kohler S, Unger T, Hoffmann S, Steinacher B, Fydrich T, Bschor T. Comparing augmentation with non-antidepressants over sticking to antidepressants after treatment failure in depression: a naturalistic study. Pharmacopsychiatry. 2013;2:69-76.

\section{Publish your work in this journal}

Neuropsychiatric Disease and Treatment is an international, peerreviewed journal of clinical therapeutics and pharmacology focusing on concise rapid reporting of clinical or pre-clinical studies on a range of neuropsychiatric and neurological disorders. This journal is indexed on PubMed Central, the 'PsycINFO' database and CAS, and is the official journal of The International Neuropsychiatric Association (INA). The manuscript management system is completely online and includes a very quick and fair peer-review system, which is all easy to use. Visit http://www.dovepress.com/testimonials.php to read real quotes from published authors. 